10,12

\title{
Размерная зависимость упругих свойств нанокристалла аргона
}

\author{
(C) М.Н. Магомедов
}

Институт проблем геотермии ДагНЦ РАН, Махачкала, Россия

E-mail: mahmag4@mail.ru

(Поступило в Редакцию 27 июня 2018 г.)

На основе RP(vac)-модели нанокристалла рассчитаны уравнение состояния $(P)$ и изотермический модуль упругости $(B)$ для макро- и нанокристалла аргона при $T=10 \mathrm{~K}$. Изучены изохорические и изобарические $($ при $P=0)$ зависимости температуры Дебая $(\Theta)$, первого $(\gamma)$ и второго $(q)$ параметров Грюнайзена, удельной поверхностной энергии $(\sigma), B$ и $B^{\prime}(P)=(\partial B / \partial P)_{T}$ от размера и формы нанокристалла. Показано, что при изотермо-изобарическом уменьшении размера нанокристалла функции $\Theta, q, \sigma, B$ и $B^{\prime}(P)$ уменьшаются, а величина $\gamma$ растет. Но в случае изотермо-изохорического уменьшения размера нанокристалла модуль упругости аргона возрастает. При отклонении формы нанокристалла от наиболее энергетически оптимальной (для $\mathrm{RP}(\mathrm{vac})$-модели это куб) размерные зависимости данных функций усиливаются.

Работа выполнена при финансовой поддержке РФФИ (гранты № 16-03-00041_a, № 18-29-11013_мк) и Программы Президиума РАН (программа № I.13).

DOI: 10.21883/FTT.2019.01.46905.175

\section{1. Введение}

Ранее в [1] нами было показано, что в области высоких температур (выше температуры Дебая: $T>\Theta$ ) при уменьшении размера (или $N$ - числа атомов) нанокристалла его изотермический модуль упругости $(B)$ уменьшается. Но в области низких температур зависимость $B(N)$ менее заметна, и возможен случай, когда функция $B(N)$ возрастает при уменьшении размера нанокристалла. Вывод об уменышении величины $B$ при уменьшении размера нанокристалла был позже получен и другими методами в работах [2,3]. Вместе с тем в литературе имеются работы (например, [4]), в которых теоретическими методами получено возрастание модуля упругости при уменьшении размера нанокристалла. Экспериментальные данные по зависимости $B(N)$ также противоречивы (см. обзор в [1-4]), что обусловлено трудностью измерения модуля упругости для нанокристаллов. При этом, если в [1] функция $B(N)$ была изучена с учетом поверхностного давления, то в [2-4] поверхностное давление не учитывалось. Поэтому осталось неясным - как ведет себя модуль упругости при изохорическом (при постоянном удельном объеме $v=V / N)$ и при изобарическом уменьшении размера нанокристалла?

Ответа на этот вопрос до настоящего времени не было ввиду отсутствия уравнения состояния нанокристалла: $P(T, v, N)$. Но недавно в [5] в рамках $\mathrm{RP}(\mathrm{vac})$-модели было получено уравнение состояния нанокристалла, учитывающее как вакансии в структуре, так и наличие делокализованных (мигрирующих по объему) атомов. Поэтому целью данной работы является изучение в рамках $\mathrm{RP}(\mathrm{vac})$-модели как изохорной $(v=\mathrm{const})$, так и изобарной $(P=0)$ зависимостей модуля упругости от размера и формы нанокристалла со свободной поверх- ностью (free standing nanocrystal). Кроме того, будет изучена и размерная зависимость функции $(\partial B / \partial P)_{P=0}$.

\section{Модель нанокристалла простого вещества}

Пусть атомы взаимодействуют посредством потенциала Ми-Леннард-Джонса [6, гл. 3]

$$
\varphi(r)=\frac{D}{(b-a)}\left[a\left(\frac{r_{0}}{r}\right)^{b}-b\left(\frac{r_{0}}{r}\right)^{a}\right],
$$

где $D$ и $r_{0}$ - глубина и координата минимума потенциала, $b>a>1$ - численные параметры.

Будем полагать, что рассматриваемая система образует решеточную структуру из $N+N_{v}$ сферических ячеек одинакового объема, из которых $N_{v}$ ячеек вакантны, а $N$ ячеек заняты тождественными сферически симметричными атомами, масса каждого из которых равна $m$. Из $N$ атомов только часть атомов $\left(N-N_{d}\right)$ локализована в ячейках, а другая часть $\left(N_{d}\right)$ - делокализована, т. е. они могут перемещаться по всему объему. Причем со временем локализованный атом, возбудившись, может стать делокализованным, и наоборот.

Для вероятности образования вакансии в решетке простого вещества было получено выражение [6,7]

$$
\phi_{v}=\frac{N_{v}}{N+N_{v}}=\frac{2}{\pi^{1 / 2}} \int_{\left[E_{v} /\left(k_{B} T\right)\right]^{1 / 2}}^{\infty} \exp \left(-t^{2}\right) d t,
$$

где $k_{B}$ - постоянная Больцмана, $E_{v}-$ энергия создания вакантного узла в решетке.

Положим, что нанокристалл со свободной поверхностью имеет вид прямоугольного параллелепипеда с 
квадратным основанием, ограненный гранями типа (100) с геометрической поверхностью Гиббса. Ячейки (как занятые, так и вакантные) образуют кристаллическую структуру с коэффициентом упаковки $k_{p}$. Величина $f=N_{p s} / N_{p o}=N_{p s}^{0} / N_{p o}^{0}$ - представляет собой параметр формы, который определяется отношением числа $N_{p s}^{0}$ атомов (или $N_{p s}=N_{p s}^{0} /\left(1-\phi_{v}\right)^{1 / 3}$ ячеек) на боковом ребре к числу $N_{p o}^{0}$ атомов (или $N_{p o}=N_{p o}^{0} /\left(1-\phi_{v}\right)^{1 / 3}$ ячеек) на ребре квадратного основания. Для стержневидной формы $f>1$, для куба $f=1$, для пластинчатой формы $f<1$.

Число ячеек и атомов в нанокристалле при данном $f$ равно

$$
N+N_{v}=f \frac{N_{p o}^{3}}{\alpha}=f \frac{\left(N_{p o}^{0}\right)^{3}}{\alpha\left(1-\phi_{v}\right)}, \quad N=f \frac{\left(N_{p o}^{0}\right)^{3}}{\alpha},
$$

где $\alpha=\pi /\left(6 k_{p}\right)$ - параметр структуры.

Ограничение системы поверхностью приведет к обрыву связей на границе. Поэтому если использовано приближение „взаимодействия только ближайших соседей“, то вместо первого координационного числа $\left(k_{n}\right)$ необходимо брать $\left\langle k_{n}\right\rangle$ - среднее (по всей наносистеме) значение первого координационного числа, которое будет зависеть как от размера, так и от формы наносистемы. При этом структуру системы полагаем неизменной: $k_{p}=$ const. Данную модель нанокристалла в виде прямоугольного параллелепипеда (Rectangular Parallelepiped), форму которого можно варьировать с помощью параметра формы $f$, назовем RP-моделью с вакансиями, или RP(vac)-моделью.

$\mathrm{B}$ рамках $\mathrm{RP}(\mathrm{vac})$-модели зависимость среднего по наносистеме значения первого координационного числа от $\alpha, N, \phi_{v}$ и $f$ имеет вид [5]

$k_{n}\left(N, \phi_{v}\right)=k_{n}^{0}(\infty)\left(1-\phi_{v}\right)\left[1-Z_{s}(f)\left(\frac{\alpha^{2}}{N}\left(1-\phi_{v}\right)\right)^{1 / 3}\right]$

где $k_{n}^{0}(\infty)$ - первое координационное число (т. е. число ближайших к данному атому занятых или вакантных ячеек) для макрокристалла.

Функция формы $Z_{s}(f)=(1+2 f) /\left(3 f^{2 / 3}\right)$, достигает минимума равного единице при $f=1$, т.е. для формы куба. Для пластинчатых $(f<1)$ или стержневидных $(f>1)$ форм значение функции формы больше единицы: $Z_{s}(f \neq 1)>1$. Поэтому функция $k_{n}(f)^{*}=k_{n}(f) / k_{n}(\infty)$ при любом значении $N+N_{v}$ имеет максимум при $f=1$, т. е. для наиболее энергетически устойчивой - кубической формы прямоугольного параллелепипеда.

Объем системы равен сумме объемов, приходящихся на одну (занятую либо вакантную) ячейку, форму которой считаем сферической

$$
V=\frac{\pi}{6 k_{p}}\left(N+N_{v}\right) c^{3}
$$

где $c=c_{0}\left(1-\phi_{v}\right)^{1 / 3}$ - расстояние между центрами ближайших ячеек, $c_{0}-$ расстояние между центрами ближайших ячеек при $N_{v}=0: c_{0}=\left[6 k_{p} V /(\pi N)\right]^{1 / 3}$.
В [5] для удельной (на атом) свободной энергии Гельмгольца было получено

$$
f_{H}=f_{i}+f_{s}+f_{w}+f_{d}+f_{k} .
$$

Здесь $f_{i}$ - это удельная свободная энергия трансляционного движения делокализованных атомов

$$
f_{i}=-x_{d} k_{B} T\left[\left(\frac{T}{A_{d}}\right)^{3 / 2} \frac{V}{V_{0}}\right],
$$

где введены обозначения

$$
A_{d}=\left(\frac{2 \pi \hbar^{2}}{m k_{B}}\right)\left(\frac{N}{e V_{0}}\right)^{2 / 3}, V_{0}=N\left(\frac{\pi}{6 k_{p}}\right) r_{0}^{3} .
$$

Здесь $\hbar$ - постоянная Планка, $e=2.718$ - основание натурального логарифма, $x_{d}$ - доля делокализованных атомов, т.е. это вероятность атома иметь кинетическую энергию выше, чем $E_{d}$ - энергии делокализации атома $[6,8]$ :

$$
x_{d}=\frac{N_{d}}{N}=\frac{2}{\pi^{1 / 2}} \int_{E_{d} /\left(k_{B} T\right)}^{\infty} t^{1 / 2} \exp (-t) d t .
$$

Второе слагаемое в (5) - это удельная свободная энергия статического взаимодействия всех атомов между собой, которая при использовании приближения „взаимодействия только ближайших соседей“ имеет вид

$$
f_{s}=\left(\frac{k_{n}}{2}\right) D \cdot U_{0}(R),
$$

где $R=r_{0} / c-$ относительная линейная плотность кристалла,

$U_{0}(R)=\frac{1}{(b-a)}\left(a R^{b}-b R^{a}\right), \quad R=\frac{r_{0}}{c}=\left[\frac{V_{0}}{V\left(1-\phi_{v}\right)}\right]^{1 / 3}$.

Третье слагаемое в (5) - это удельная свободная энергия колебательного движения локализованных атомов по модели кристалла Эйнштейна $\left(\Theta_{E}\right.$ - температура Эйнштейна)

$$
f_{w}=3\left(1-x_{d}\right) k_{B} T\left\{0.5 \frac{\Theta_{E}}{T}+\ln \left[1-\exp \left(-\frac{\Theta_{E}}{T}\right)\right]\right\} .
$$

Член $f_{d}$ - это удельная свободная энергия динамического взаимодействия делокализованных атомов из-за их смещения от центров ячеек при миграции по объему $[6$, стр. 21]

$$
f_{d}=\frac{x_{d} k_{n} a b D}{2(b-a)}\left\{R^{b}\left[\frac{l_{3}\left(b, \xi_{p}\right)}{b}\right]-R^{a}\left[\frac{l_{3}\left(a, \xi_{p}\right)}{a}\right]\right\},
$$

где введены обозначения

$$
\begin{gathered}
l_{3}(k, t)=\frac{(1+t)^{k-2}-(1-t)^{k-2}}{2(k-2) t\left(1-t^{2}\right)^{k-2}}-1, \\
\xi_{p}=\frac{0.5}{3^{1 / 2} k_{p}^{1 / 3}}<0.5 .
\end{gathered}
$$


Последнее, входящее в (5) слагаемое $f_{k}-$ это удельная свободная энергия „перестановочного движения“ атомов, которая возникает из-за применения формулы Стирлинга. Оно исчезает в „термодинамическом пределе“, т.е. при $N \rightarrow \infty, V \rightarrow \infty$ и $v=V / N=$ const [6, стр. 23]

$$
f_{k}=-\frac{k_{B} T}{N} \ln \left[\frac{\left(N_{d} / e\right)^{N_{d}}}{N_{d} !}\right] \cong \frac{k_{B} T}{2 N} \ln \left(2 \pi N_{d}\right) .
$$

Входящие в (2)-(14) функции $E_{v}, E_{d}$ и $\Theta_{E}$ были определены в работах [6-9]. Энергия создания электронейтральной вакансии в структуре кристалла имеет вид $[6,7]$

$$
E_{v}=\frac{E_{L}}{1+x_{d}\left[\left(C_{D} E_{L} / k_{B} T\right)-1\right]},
$$

где введены обозначения

$$
\begin{gathered}
E_{L}=\frac{m}{k_{n}^{0}}\left(\frac{c_{0} k_{B} \Theta_{E_{0}}}{2 \hbar}\right)^{2} f_{y}\left(\frac{\Theta_{E_{0}}}{T}\right), \quad C_{D}=\frac{4 k_{n}^{0}}{3 k_{p}^{2 / 3}}, \\
f_{y}\left(y_{w}\right)=\frac{2}{y_{w}} \frac{[1-\exp (-y w)]}{\left[1+\exp \left(-y_{w}\right)\right]} .
\end{gathered}
$$

Индекс „, $0^{\star}$ у функций $k_{n}^{0}, c_{0}$ и $\Theta_{E_{0}}$ означает, что функции рассчитаны для исходной (не срелаксировавшей в активированное вакансиями состояние) безвакансионной виртуальной решетки (т. е. при $\left.\phi_{v}=0\right)$.

Для энергии делокализации атома в объеме кристалла было получено выражение $[6,8]$

$$
E_{d}=\left(\frac{27}{128 \pi^{2}}\right) m\left(\frac{c_{0} k_{B} \Theta_{0}}{\hbar k_{p}^{1 / 3}}\right)^{2} f_{y}\left(\frac{3 \Theta_{0}}{4 T}\right),
$$

Здесь $\Theta$ - температура Дебая, которая связана с температурой Эйнштейна соотношением: $\Theta=(4 / 3) \Theta E$. Для функции $\Theta$ было получено выражение $[6,9]$

$$
\Theta=A_{w} \xi\left[-1+\left(1+\frac{8 D}{k_{B} A_{w} \xi^{2}}\right)^{1 / 2}\right],
$$

где введены следующие обозначения:

$$
\begin{gathered}
\xi=\frac{9}{k_{m}^{0}(\infty)}, A_{w}=K_{R} \frac{5 k_{n} a b(b+1)}{144(b-a)}\left(\frac{r_{0}}{c}\right)^{b+2}, \\
K_{R}=\frac{\hbar^{2}}{k_{B} r_{0}^{2} m} .
\end{gathered}
$$

\section{2. Делокализация атомов на свободной поверхности Гиббса}

B RP(vac)-модели локализованный атом колеблется в ячейке, образованной ближайшими соседями. При этом для описания колебаний локализованных атомов используется модель Эйнштейна, т. е. колебательное движение локализованного атома рассматривается как колебания трех независимых гармонических осциллятора с одинаковой частотой. Но для атома, колеблющегося на поверхности Гиббса, некоторые направления являются свободными для ухода из ячейки. Поэтому движения в этих направлениях следует описывать не как движения гармонического осциллятора, а как движение делокализованного атома.

B рамках RP(vac)-модели число ячеек (как занятых, так и свободных), лежащих на поверхности равно $[6,10]$

$$
N_{s}=4 N_{s s}+2 N_{s o}+8 N_{f o}+4 N_{f s}+8 .
$$

Здесь $N_{s s}=N_{f o} N_{f s}$ и $N_{s o}=N_{f o}^{2}$ - число ячеек на поверхности боковой грани и грани основания (без учета ячеек на ребрах и вершинах); $N_{f s}=N_{p s}-2$ и $N_{f o}=N_{p o}-2$ - число ячеек на боковом ребре и на ребре основания (без учета ячеек в вершинах) параллелепипеда. Тогда для RP(vac)-модели получим

$$
N_{s}=2\left[(2 f+1) N_{p o}^{2}-2(f+2) N_{p o}+4\right] .
$$

При этом число занятых ячеек (т.е. атомов) на поверхности Гиббса будет равно: $N_{s}^{o}=N_{s}\left(1-\phi_{v}\right)$. Из них локализованными по объемному закону (8) будет следующее число атомов:

$$
N_{s l}^{o}=N_{s}^{o}\left(1-x_{d v}\right)=N_{s}\left(1-\phi_{v}\right)\left(1-x_{d v}\right),
$$

где $x_{d v}$ - доля делокализованых атомов в объеме, которая рассчитывается по формулам (8) и (17), а функция $\phi_{v}$ рассчитывается по формулам (2), (15) и (16) при использовании значения $x_{d v}$, определенного для объема.

На поверхности боковой грани и грани основания из колебаний локализованного атома нужно исключить один гармонический осциллятор из трех, так как в этом, перпендикулярном грани, направлении делокализация будет происходить уже по иному, отличному от „объемного“, способу. Для атома на боковом ребре и на ребре основания по аналогичным причинам нужно исключить два гармонических осциллятора, ибо гармонические колебания в ячейке совершает только тот осциллятор, который колеблется вдоль ребра. По этим же причинам следует считать, что атом в вершине делокализуется по отличному от „объемного“ способа. Поэтому среди локализованных на поверхности Гиббса атомов некоторую часть $\left(N_{d s}\right)$ следует считать делокализующимися по отличному от „объемного“ способа. В рамках $\mathrm{RP}(\mathrm{vac})-$ модели для $N_{d s}$ можно получить

$$
\begin{aligned}
& N_{d s}=\left[\left(4 N_{s s}+2 N_{s o}\right)(1 / 3)+\left(8 N_{f o}+4 N_{f s}\right)(2 / 3)+8\right] \\
& \times\left(1-\phi_{v}\right)\left(1-x_{d v}\right)=(2 / 3)(2 f+1) N_{p o}^{2}\left(1-\phi_{v}\right)\left(1-x_{d v}\right) \\
& =(2 / 3)(2 f+1)\left(N_{p o}^{0}\right)^{2}\left(1-\phi_{v}\right)^{1 / 3}\left(1-x_{d v}\right) .
\end{aligned}
$$

Пусть $x_{d s}$ - это вероятность делокализации атома на поверхности Гиббса способом, отличным от того, каким атом делокализуется в объеме. Тогда для доли общего числа делокализованных атомов в наносистеме получим

$$
x_{d}=\frac{\left(N-N_{d s}\right) x_{d v}+N_{d s} x_{d s}}{N}=\left(1-\eta_{d s}\right) x_{d v}+\eta_{d s} x_{d s},
$$


где доля атомов, делокализованных на поверхности Гиббса по отличному от „объемного“ способа, равна

$$
\begin{aligned}
\eta_{d s}=\frac{N_{d s}}{N} & =\frac{2(2 f+2)}{3 N}\left(N_{p o}^{0}\right)^{2}\left(1-\phi_{v}\right)^{1 / 3}\left(1-x_{d v}\right) \\
& =\frac{2(2 f+2)}{3 f N_{p o}^{0}}\left(1-\phi_{v}\right)^{1 / 3}\left(1-x_{d v}\right) \\
& =2 \frac{Z_{s}(f)}{(\alpha N)^{1 / 3}}\left(1-\phi_{v}\right)^{1 / 3}\left(1-x_{d v}\right) .
\end{aligned}
$$

Пусть делокализация атома со свободной поверхности Гиббса связана с переходом атома в газовую фазу и возвратом атома обратно (ибо наложено условие $N=$ const). Тогда для величины энергии делокализации атома в формуле (8) можно принять: $E_{d s}=m\langle v\rangle^{2} / 2$, где $\langle v\rangle$ - некоторая, характерная для газовой среды, скорость атома. Если брать $\langle v\rangle$ как наиболее вероятную скорость, или как среднеквадратичную скорость атомов в газе $\left(\langle v\rangle=\left(3 k_{B} T / m\right)^{1 / 2}\right) \quad[11$, стр. 117], то можно получить: $E_{d s} /\left(k_{B} T\right)=1$ (если $\langle v\rangle$ это наиболее вероятная скорость атомов) или 1.5 (если $\langle v\rangle$ это среднеквадратичная скорость атомов). Поэтому в соответствии с (8), величина $x_{d s}$ будет равна постоянной величине: $x_{d s}=0.392$ (если $\langle v\rangle$ это среднеквадратичная скорость атомов), $x_{d s}=0.572$ (если $\langle v\rangle$ это наиболее вероятная скорость атомов). Поэтому для простоты можно принять: $x_{d s}=0.5$.

\section{3. Результаты расчета для нанокристалла аргона}

Для расчетов был взят аргон ( $m(\mathrm{Ar})=39.95$ a.m.u.), имеющий в твердой фазе гранецентрированную кубическую (ГЦК) структуру: $k_{n}^{0}(\infty)=12, k_{p}=0.7405$. Параметры парного межатомного потенциала Ми-ЛеннардДжонса (1) были определены в [6] самосогласованным образом из экспериментальных данных ГЦК-Ar, полученных при $T=0 \mathrm{~K}, P=0$. Они имеют следующие значения:

$r_{0}=3.7555 \cdot 10^{-10} \mathrm{~m}, D / k_{B}=173.6 \mathrm{~K}, a=6, b=17$.

Нормированное уравнение состояния было получено путем численного дифференцирования изотермической зависимости функции $f_{H} / D$ по нормированному объему: $P v_{0} / D=-\left[\partial\left(f_{H} / D\right) / \partial\left(v / v_{0}\right)\right]_{T}$.

Изотермический модуль упругости рассчитывался путем численного дифференцирования изотермической зависимости функции $P$ по нормированному объему: $B=-\left(v / v_{0}\right)\left[\partial P / \partial\left(v / v_{0}\right)\right]_{T}$.

На рис. 1 показаны результаты расчета изотермоизоморфных (т.е. при постоянных значениях $T=10 \mathrm{~K}$ и $f$ ) зависимостей нормированного уравнения состояния для ГЦК-Ar от нормированного объема. На рис. 2 и 3 показаны результаты расчета изотермоизоморфных зависимостей модуля упругости от нормированного объема (рис. 2), и от нормированного давления (рис. 3). Сплошные линии получены при $f=1$, т.е. для кубической формы кристалла. Пунктирные линии получены при $f=8$, т.е. для нанокристалла стержневидной формы. Пунктирная линия на рис. 1 это экспериментальная зависимость, полученная в $[12$, Eq. (9)] для макрокристалла ГЦК-Ar при $T=4.2 \mathrm{~K}$. На рис. $1-3$ сплошная линия 1 получена для макрокристалла $\left(N_{p o}^{0}=10^{8}\right.$, т. е. $\left.N=1.4 \cdot 10^{24}\right)$. Линии 2 и 3 получены при $N=f\left(N_{p o}^{0}\right)^{3} / 0.707=11315$ : сплошная линия 2 получена для куба при $N_{p o}^{0}=20$ и $f=1$, а пунктирная линия 3 получена для стержня при $N_{p o}^{0}=10$ и $f=8$. Линии 4 и 5 получены при $N=f\left(N_{p o}^{0}\right)^{3} / 0.707=305$ : сплошная линия 4 получена для куба при $N_{p o}^{0}=6$ и $f=1$, а пунктирная линия 5 получена для стержня при $N_{p o}^{0}=3$ и $f=8$.

Из рис. 1 видно, что наши расчеты для макрокристалла хорошо согласуются с экспериментальными данными из [12]. При этом надо учесть, что наши данные получены при $T=10 \mathrm{~K}$, поэтому они лежат чуть выше данных из [12], которые получены при $T=4.2 \mathrm{~K}$. При изоморфном уменьшении размера давление в нанокристалле возрастает, что обусловлено вкладом поверхностного давления. Причем, чем больше форма нанокристалла отклонена от наиболее энергетически оптимальной кубической формы, тем резче происходит рост давления на изотерме.

Из рис. 2 и 3 видно, что при изотермо-изоморфном уменьшении числа атомов в нанокристалле ГЦК-Ar модуль упругости возрастает если процесс изохорический (см. рис. 2) и уменьшается в случае изобарического процесса (рис. 3). Причем, чем больше форма нанокристалла отклонена от кубической формы, тем заметнее происходит изменение модуля упругости при уменьшении размера.

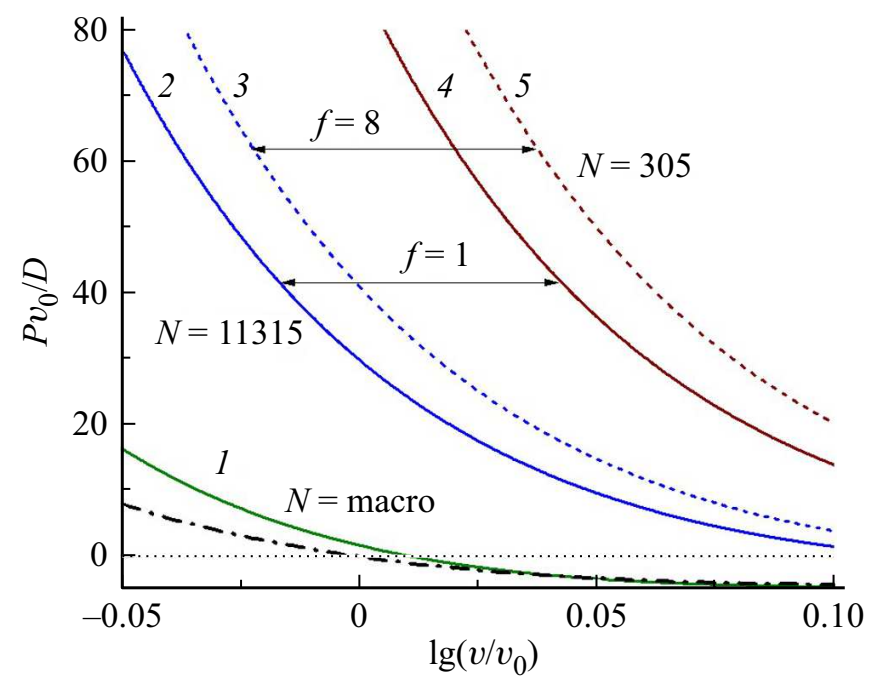

Рис. 1. Изотермо-изоморфные (т. е. при постоянных значениях $T=10 \mathrm{~K}$ и $f$ ) зависимости нормированного уравнения состояния ГЦК-Аr от нормированного объема. Сплошные линии рассчитаны при $f=1$, пунктирные линии - при $f=8$. 
Таблица 1. Значения температуры Дебая, первого и второго параметров Грюнайзена, удельной поверхностной энергии грани (100) и изотермического модуля упругости, рассчитанные для изотермо-изохорического $\left(T=10 \mathrm{~K}, R=r_{0} / c=1\right)$ уменьшения размера или деформации формы нанокристалла ГЦК-Ar

\begin{tabular}{c|c|c|c|c|c|c|c}
\hline$N_{p o}^{0}$ & $f$ & $N$ & $\Theta(R=1), \mathrm{K}$ & $\gamma(R=1)$ & $q(R=1)$ & $\sigma(R=1), 10^{-3} \mathrm{~J} / \mathrm{m}^{2}$ & $B(R=1), \mathrm{GPa}$ \\
\hline $10^{8}$ & 1 & Macro & 86.81 & 3.011 & 0.163 & 20.46 & 4.77 \\
20 & 1 & 11315 & 85.33 & 3.014 & 0.160 & 2.77 & 16.73 \\
10 & 8 & $-{ }^{\prime \prime}-$ & 84.71 & 3.015 & 0.159 & -4.61 & 21.56 \\
6 & 1 & 305 & 81.78 & 3.020 & 0.153 & -38.51 & 41.31 \\
3 & 8 & $-^{\prime \prime}-$ & 79.57 & 3.024 & 0.149 & -63.07 & 53.83
\end{tabular}

Таблица 2. Значения температуры Дебая, первого и второго параметров Грюнайзена, удельной поверхностной энергии грани $(100)$ и изотермического модуля упругости, рассчитанные для изотермо-изобарического $(T=10 \mathrm{~K}, P=0$ или $2 \mathrm{GPa})$ уменьшения размера или деформации формы нанокристалла ГЦК-Ar

\begin{tabular}{c|c|c|c|c|c|c|c|c|c}
\hline$N_{p o}^{0}$ & $f$ & $N$ & $\begin{array}{c}\Theta(P=0) \\
\mathrm{K}\end{array}$ & $\gamma(P=0)$ & $q(P=0)$ & $\begin{array}{c}\sigma(P=0) \\
10^{-3} \mathrm{~J} / \mathrm{m}^{2}\end{array}$ & $\begin{array}{c}B(P=0) \\
\mathrm{GPa}\end{array}$ & $\begin{array}{c}B(P=2 \mathrm{GPa}) \\
\mathrm{GPa}\end{array}$ & $\Delta B / \Delta P$ \\
\hline $10^{8}$ & 1 & Macro & 80.33 & 3.023 & 0.150 & 20.10 & 3.75 & 20.05 & 8.15 \\
20 & 1 & 11315 & 36.83 & 3.102 & 0.066 & 10.71 & 1.76 & 17.42 & 7.83 \\
10 & 8 & $-{ }^{\prime \prime}-$ & 30.47 & 3.114 & 0.054 & 9.29 & 1.48 & 17.02 & 7.77 \\
6 & 1 & 305 & 17.62 & 3.136 & 0.031 & 6.37 & 0.93 & 16.04 & 7.56 \\
3 & 8 & $-{ }^{\prime \prime}-$ & 14.15 & 3.142 & 0.025 & 5.50 & 1.07 & 15.69 & 7.31
\end{tabular}

В табл. 1 представлены рассчитанные для изотермоизохорического $\left(T=10 \mathrm{~K}, R=r_{0} / c=1\right)$ процесса: температура Дебая $(\Theta)$, первый $\left(\gamma=-[\partial \ln (\Theta) / \partial \ln (v)]_{T}\right)$ и второй $\left(q=[\partial \ln (\gamma) / \partial \ln (v)]_{T}\right)$ параметры Грюнайзена, удельная (на единицу площади) поверхностная энергия грани (100) и модуль упругости $(B)$. Поверхностная энергия в рамках $\mathrm{RP}(\mathrm{vac})$-модели была рассчитана по методу из [5]. Помимо макрокристалла данные функции были рассчитаны и для нанокристаллов из 11315 и

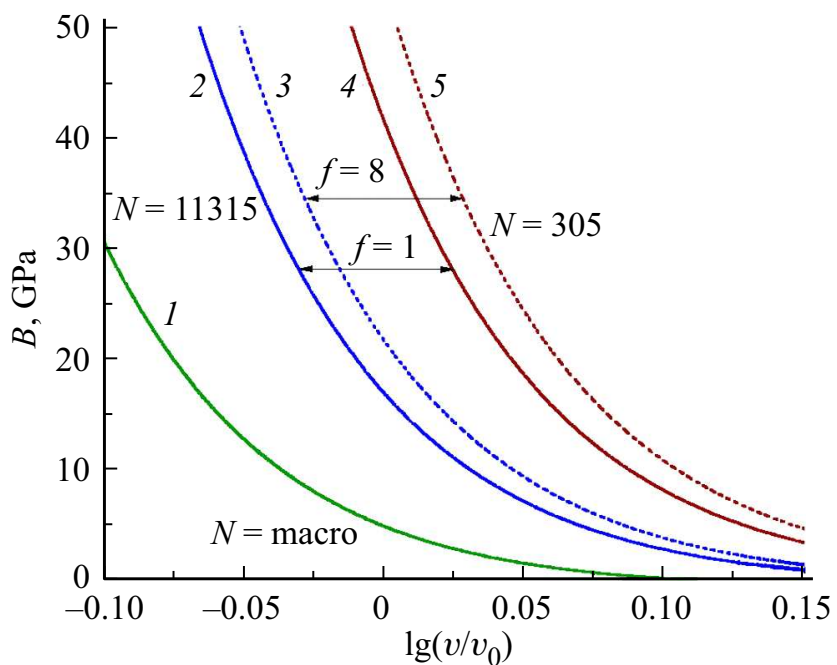

Рис. 2. Изотермо-изоморфные (при $T=10 \mathrm{~K}$ и $f=1$ или 8 ) зависимости модуля упругости ГЦК-Аr от нормированного объема. Сплошные линии рассчитаны при $f=1$, пунктирные линии - при $f=8$.

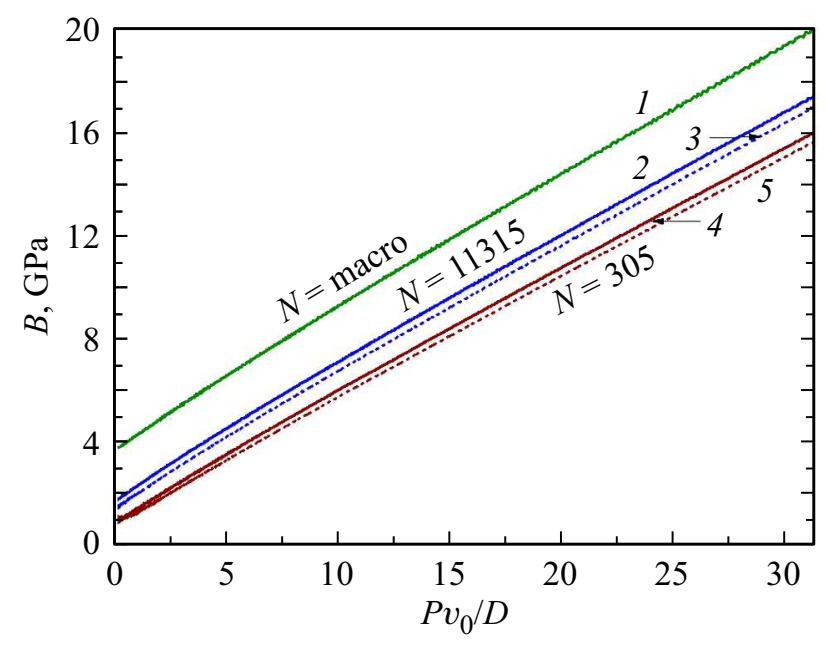

Рис. 3. Изотермо-изоморфные (при $T=10 \mathrm{~K}$ и $f=1$ или 8) зависимости модуля упругости ГЦК-Аr от нормированного давления. Сплошные линии рассчитаны при $f=1$, пунктирные линии - при $f=8$.

из 305 атомов. При этом для каждого числа атомов расчеты выполнены для нанокристалла кубической формы $\left(N_{p o}^{0}=20\right.$ и $N_{p o}^{0}=6$ при $\left.f=1\right)$ и стержневидного нанокристалла $\left(N_{p o}^{0}=10\right.$ и $N_{p o}^{0}=3$ при $\left.f=8\right)$.

В табл. 2 представлены значения данных функций рассчитанные для изотермо-изобарического $(T=10 \mathrm{~K}$, $P=0)$ процесса. Там же представлены значения $B$, полученные для изобары $P=2 \mathrm{GPa}$, по которым было рассчитано изменение наклона зависимости $B(P)$ для 
нанокристаллов различных размеров и форм по формуле

$$
\Delta B / \Delta P \cong B^{\prime}(P, T)=(\partial B / \partial P)_{T} .
$$

Экспериментальные оценки данных функций для макрокристалла аргона лежат в интервале:

$\Theta(P=0,10 \mathrm{~K}) /[\mathrm{K}]=85-93.3$ [6, стр. 45], $92[13]$, 85-95 [14];

$\gamma(P=0,10 \mathrm{~K})=2.4-3.1[6$, стр. 45], $2.73[12], 3.2[13]$, $2.3-2.9[14]$;

$\sigma(P=0,10 \mathrm{~K}) /\left[10^{-3} \mathrm{~J} / \mathrm{m}^{2}\right]=20.39 \quad[6$, стр. 428; 15], $28.5[16]$

$B(P=0,10 \mathrm{~K}) /[\mathrm{GPa}]=2.86[12], 3.8[13], 2.5-3.2[14]$;

$B^{\prime}(P=0, T=10 \mathrm{~K})=7.2[12], 8.3[13]$.

Из табл. 2 видно, что согласие наших расчетов для макрокристалла ГКЦ-Ar с экспериментальными оценками вполне хорошее. Отметим, что расчеты параметров образования решеточных вакансий и самодиффузии атомов для макрокристалла аргона были проведены нами в $[6$, стр. $304 ; 17]$ и также показали хорошее согласие с экспериментальными оценками.

Из табл. 1 видно, что при изотермо-изохорноизоморфном уменьшении размера функции $\Theta, q$ и $\sigma$ уменьшаются, а величины $B$ и $\gamma$ растут. При $N=11315$ и при $f=8$ или при $N=305$ и при любом $f$ нанокристалл имеет отрицательную поверхностную энергию, что должно приводить к фрагментации нанокристалла.

Из табл. 2 видно, что при изотермо-изобарноизоморфном уменьшении размера функции $\Theta, q, \sigma, B$ и $B^{\prime}(P)$ уменьшаются, а величина $\gamma$ растет. При этом изменения функций $\Theta, \gamma$ и $q$ происходят значительно заметнее, чем при изохорическом уменьшении размера.

\section{4. Выводы}

На основе $\mathrm{RP}(\mathrm{vac})$-модели рассчитано уравнение состояния аргона при $T=10 \mathrm{~K}$ как в макро-, так и в нанокристаллическом состоянии. Показано, что изоморфное уменьшение размера нанокристалла вызывает рост давления, что обусловлено вкладом поверхностного давления. Причем, чем больше форма нанокристалла отклонена от наиболее энергетически оптимальной формы (для RP(vac)-модели это куб), тем резче происходит рост давления на изотерме.

Показано, что при изотермо-изохорно-изоморфном уменьшении размера нанокристалла функции $\Theta, q$ и $\sigma$ уменьшаются, а величины $\gamma$ и $B$ увеличиваются. Возрастание модуля упругости при данном процессе обусловлено ростом давления при уменьшении размера нанокристалла.

При изотермо-изобарно-изоморфном уменьшении размера нанокристалла функции $\Theta, q, \sigma, B$ и $B^{\prime}(P)$ уменьшаются, а величина $\gamma$ растет. Уменьшение модуля упругости связано с необходимостью растяжения нанокристалла для выравнивания давления до $P=0$.

При изотермо-изобарно-изоморфном уменьшении размера нанокристалла функции $\Theta, q$ и $\gamma$ изменяются больше, а функция $\sigma$ уменьшается меньше, чем в случае изотермо-изохорно-изоморфного уменьшения размера.
При отклонении формы нанокристалла от наиболее энергетически оптимальной формы (для $\mathrm{RP}(\mathrm{vac})$-модели это куб) размерные зависимости функций $\Theta, q, \gamma, \sigma, B$ и $B^{\prime}(P)$ усиливаются, как для изотермо-изохорического, так и для изотермо-изобарического процесса.

Таким образом, полученное в разных теоретических или экспериментальных работах различие в зависимости модуля упругости от размера нанокристалла может быть обусловлено характером процесса, при котором происходило изотермо-изоморфное уменьшение размера нанокристалла в этих работах.

Автор благодарен Э.Н. Ахмедову, Н.Ш. Газановой и 3.М. Сурхаевой за помощь в работе и полезные дискуссии.

\section{Список литературы}

[1] М.Н. Магомедов. Письма в ЖТФ 39, 9, 9 (2013). DOI: $10.1134 / \mathrm{S} 1063785013050076$

[2] L. Liang, M. Li, F. Qin, Y. Wei. Philosoph. Mag. 93, 6, 574 (2013). DOI: 10.1080/14786435.2012.725950

[3] C.J. Bhatt, K. Kholiya. Indian J. Pure Appl. Phys. 52, 9, 604 (2014). http://nopr.niscair.res.in/handle/123456789/29354

[4] R. Cherian, C. Gerard, P. Mahadevan, N.T. Cuong, R. Maezono. Phys. Rev. B 82, 23, 235321 (2010).

DOI: $10.1103 /$ PhysRevB.82.235321

[5] М.Н. Магомедов. Поверхность. Рентген., синхротр., и нейтрон. исслед. 2, 103 (2018). DOI: $10.1134 / \mathrm{S} 1027451018010299$

[6] М.Н. Магомедов. Изучение межатомного взаимодействия, образования вакансий и самодиффузии в кристаллах. Физматлит, М. (2010). 544 с.

[7] М.Н. Магомедов. ФТП 42, 10, 1153 (2008). DOI: $10.1134 / \mathrm{S} 1063782608100011$

[8] М.Н. Магомедов. ФТП 44, 3, 289 (2010). DOI: $10.1134 / \mathrm{S} 1063782610030012$

[9] М.Н. Магомедов. ФТТ 45, 1, 33 (2003). DOI: $10.1134 / 1.1537405$

[10] М.Н. Магомедов. ФТТ 45, 7, 1159 (2003). DOI: $10.1134 / 1.1594232$

[11] Дж. Гиршфельдер, Ч. Кертисс, Р. Берд. Молекулярная теория газов и жидкостей. Изд-во ИЛ, М. (1961). 931 с. [J.O. Hirschfelder, Ch.F. Curtiss, B.B. Bird. Molecular Theory of Gases and Liquids. J. Wiley and Sons Ltd., N.Y. (1954). 1249 p.]

[12] M.S. Anderson, C.A. Swenson. J. Phys. Chem. Solids 36, 2, 145 (1975). DOI: 10.1016/0022-3697(75)90004-9

[13] W.B. Holzapfel, M. Hartwig, G. Reiß. J. Low Temp. Phys. 122, 3-4, 401 (2001). DOI: 10.1023/A:1004865204631

[14] V.Yu. Bodryakov. Open Sci. J. Mod. Phys. 2, 6, 111 (2015).

[15] М.Н. Магомедов. Поверхность. Рентген., синхротр., и нейтрон. исслед. 7, 103 (2013). DOI: $10.1134 / \mathrm{S} 1027451013030087$

[16] А.Х. Кяров, А.И. Темроков, Б.В. Хаев. Теплофизика высоких температур 35, 3, 386 (1997).

[17] М.Н. Магомедов. Письма в ЖТФ 27, 18, 36 (2001). DOI: $10.1134 / 1.1407355$

Редактор Т.Н. Василевская 